\title{
Mechanistic insights into the efficacy of cell penetrating peptide-based cancer vaccines
}

\author{
Morgan Grau' ${ }^{1}$ Paul R. Walker² Madiha Derouazi $^{1}$
}

Received: 6 July 2017 / Revised: 15 November 2017 / Accepted: 22 February 2018 / Published online: 5 March 2018

(c) The Author(s) 2018. This article is an open access publication

\begin{abstract}
Immunotherapies are increasingly used to treat cancer, with some outstanding results. Immunotherapy modalities include therapeutic vaccination to eliminate cancer cells through the activation of patient's immune system against tumor-derived antigens. Nevertheless, the full potential of therapeutic vaccination has yet to be demonstrated clinically because many early generation vaccines elicited low-level immune responses targeting only few tumor antigens. Cell penetrating peptides (CPPs) are highly promising tools to advance the field towards clinical success. CPPs efficiently penetrate cell membranes, even when linked to antigenic cargos, which can induce both CD8 and CD4 T-cell responses. Pre-clinical studies demonstrated that targeting multiple tumor antigens, even those considered to be poorly immunogenic, led to tumor regression. Therefore, CPP-based cancer vaccines represent a flexible and powerful means to extend therapeutic vaccination to many cancer indications. Here, we review recent findings in CPP development and discuss their use in next generation immunotherapies.
\end{abstract}

Keywords Cell penetrating peptides $\cdot$ Cancer vaccines $\cdot$ Immunotherapy $\cdot$ Tumor antigens $\cdot$ Antigen processing

\section{Introduction}

Cell penetrating peptides (CPPs) are peptides of 8-40 residues that have the ability to cross the cell membrane and enter into most cell types. Alternatively, they are also called protein transduction domain (PTDs) reflecting their origin as natural proteins. It is now more since 20 years since Frankel and Pabo as well as Green and Lowenstein described the ability of the trans-activating transcriptional activator from the human immunodeficiency virus 1 (HIV-TAT) to penetrate into cells [1]. In 1991, transduction into neural cells of the Antennapedia homeodomain (DNA-binding domain) from Drosophila melanogaster was described [2]. In 1994, the first 16-mer CPP called Penetratin (RQIKIYFQNRRMKWKK)

Madiha Derouazi

madiha.derouazi@amaltherapeutics.com

Morgan Grau

morgan.grau@amaltherapeutics.com

Paul R. Walker

Paul.Walker@unige.ch

Amal Therapeutics, Geneva, Switzerland

2 Center for Translational Research in Onco-Hematology, Division of Oncology, Geneva University Hospitals and University of Geneva, Geneva, Switzerland was characterized from the third helix of the homeodomain of Antennapedia [3], followed in 1998 by the identification of the minimal domain of TAT (YGRKKRRQRRR) required for protein transduction [4]. More recently, other peptides of different origin were described; these include viral proteins (e.g., VP22 [5] and ZEBRA [6]), or those present in venoms (e.g., melittin [7], mastoporan [8], maurocalcin [9], crotamine [10] or buforin [11]). Synthetic CPPs were also designed including the polyarginine (R8, R9, R10 and R12) [12] or transportan [13]. All of these CPPs can be classified into three different categories: (1) cationic CPPs such as TAT, penetratin, and poly-arginine, in which the positive charge relies principally on arginine and lysine residues; (2) amphipathic CPPs such as Transportan and Pep-1, where the cationic residues are separated by hydrophobic residues and the positive charge relies principally on lysine residues; (3) hydrophobic CPPs [14]. The different classes of CPPs have been extensively reviewed elsewhere [14].

Over the past 20 years, multiple applications and uses of CPPs have been described. These very versatile and promising vectors were used for intracellular delivery of a wide range of cargos, such as small molecules, nucleic acids, peptides and proteins. In many cases this is in the context of drug delivery in the field of oncology. In this review, we will describe applications of CPPs to cancer immunotherapy, with particular emphasis on cancer vaccines. To understand 
the rationale and the critical factors for efficacious use, we will discuss several important notions, such as the internalization route followed by the CPP-cargo, the nature of antigenic cargos, and the choice of adjuvants.

\section{CPPs coupled to antigenic cargos and their application to cancer vaccines}

Cell penetrating peptides are well-suited to deliver antigenic peptides or proteins to induce adaptive immune responses [15]. Although most vaccines for infectious diseases also achieve this, the response of protein or inactivated viral vaccines is biased towards CD4 T cells and neutralizing antibodies. For cancer vaccines, induction of an integrated immune response including a CD8 T-cell component is considered essential. Dendritic cells (DCs) are professional antigen presenting cells (APCs) able to activate both CD8 and CD4 T cells by presenting captured$\mathrm{Ag}$ in association with MHC-I or MHC-II molecules, respectively; CPPs can facilitate this key immunological mechanism [15]. We demonstrated the feasibility of this approach using a CPP (Z12) derived from EBV ZEBRA protein linked to a long OVA derived peptide containing the CD8-specific OVA $257-264$ epitope [16]. Vaccination of mice with a very low dose $(10 \mathrm{nM})$ of this construction and (adjuvanted with anti-CD40 antibody and Hiltonol) led to the generation of a strong OVA-specific CD8 T-cell response, as revealed by $\mathrm{OVA}_{257-264}$ dextramer staining. In contrast, when vaccination was undertaken with the same dose of OVA long peptide antigen without CPP, immune response induction was negligible. OVA-specific CD8 T-cell immune responses have also been observed by linking the model ovalbumin to other CPPs such as TAT [17, 18], Penetratin $[19,20]$ and the translocation motif of HBV [21]. Moreover, immunization with TAT fused to OVA or HPV-E7 resulted in long-term protection in tumor re-challenge experiments, indicating memory response induction [22, 23].

Eliciting immune responses to tumor-associated $\mathrm{Ag}$ (TAAs) that are also self $\mathrm{Ag}$ is particularly challenging, since such Ag are generally poorly immunogenic due to central tolerance. We showed that vaccination of mice with Z12 or other ZEBRA-derived CPPs (Z13 and Z14) linked to the gp100 TAA/self Ag led to the generation of a gp100-specific CD8 T-cell response $[16,24]$. Accordingly, we observed that Z14-gp100 therapeutic vaccination of mice injected iv with B16 cancer cells expressing gp100 leads to a near twofold reduction in the number of detectable lung metastases. In the same experimental system, we measured more than threefold reduction of lung metastasis in mice vaccinated with Z13 linked to another TAA/self Ag, TRP2 [24]. These series of observations demonstrate that the ZEBRA-derived CPP-linked to antigenic cargo is a very powerful system able to break self-tolerance and to induce therapeutic anti-tumor immune responses in vivo. Increased Ag-specific immune responses have also been reported by linking other tumor $\mathrm{Ag}$ to a CPP, such as TRP2 [25-27], carcinoembryonic antigen (CEA) [28], p53 [29], survivin [30], MUC-1 [31], HPV16 E7 [23], or HER2/neu [32]. Moreover, vaccination with these constructs induced either prophylactic or therapeutic antitumor effects in vivo. Considering that these $\mathrm{Ag}$ are often less immunogenic than model antigens such as OVA, all these observations support the use of CPPs as cancer vaccine vectors. One potential mechanism that can explain the efficacy of CPP-based cancer vaccines might be increased presentation of antigenic peptides on MHC molecules on the surface of APCs. In this regard, Wang et al. demonstrated that linking TRP2 $\mathrm{Ag}$ to CPP1 prolongs Ag presentation by DC [33]. Indeed, DC transduced with CPP-TRP2 stimulated TRP2-specific T cells in vitro for much longer periods than TRP2-pulsed DC. Another potential explanation would be that unlike proteinbased vaccines, CPP-based vaccine might be more efficient at targeting of $\mathrm{Ag}$ processing and presentation machinery.

\section{Antigen-presentation pathways followed by CPP-Ag cargos}

Although some direct plasma membrane penetration can occur, especially at the high CPP concentrations reached in some experimental systems [34], it has been demonstrated that the main translocation mechanism used by CPP-cargo constructions is the endocytic pathway (for review, see Ref. [35]) (Fig. 1). In the context of therapeutic cancer vaccines, the mechanism of CPP-cargo entry plays a central role for antigen delivery and subsequent presentation. Cytosolic proteins will be processed and presented on MHC class I molecules, whereas exogenous proteins taken up by endocytosis will be processed and presented by MHC class II molecules (Fig. 1). However, certain DC subsets can also cross present exogenous Ag on MHC-I molecules [36]. To ensure efficient MHC-I presentation of CD8-specific TAAs, antigenic cargos linked to CPP and trapped in endosomes have to reach the cross presentation pathway.

Classical cross presentation involves a proteasomedependent Ag degradation step that occurs in the cytoplasm. Thus, if $\mathrm{Ag}$ enter the endosomes, they must subsequently escape to enter this cross presentation pathway. Indeed, DC subsets can release endosome-trapped Ag into the cytosol, a process thought to involve the recruitment of endoplasmic reticulum (ER)-molecules, such as SEC61 and p97 ERAD proteins, to the endosome compartment [36]. However, it was demonstrated that certain CPPs can promote Ag endosome escape (Fig. 1). By comparing three CPPs linked to OVA, Mitsui et al. showed that the R9-OVA cancer vaccine is efficient at inducing OVA-specific immune responses in vitro and in vivo, leading to a strong anti-tumor effect [37]. When coupled to OVA, the CPP LAH4 induces tenfold 


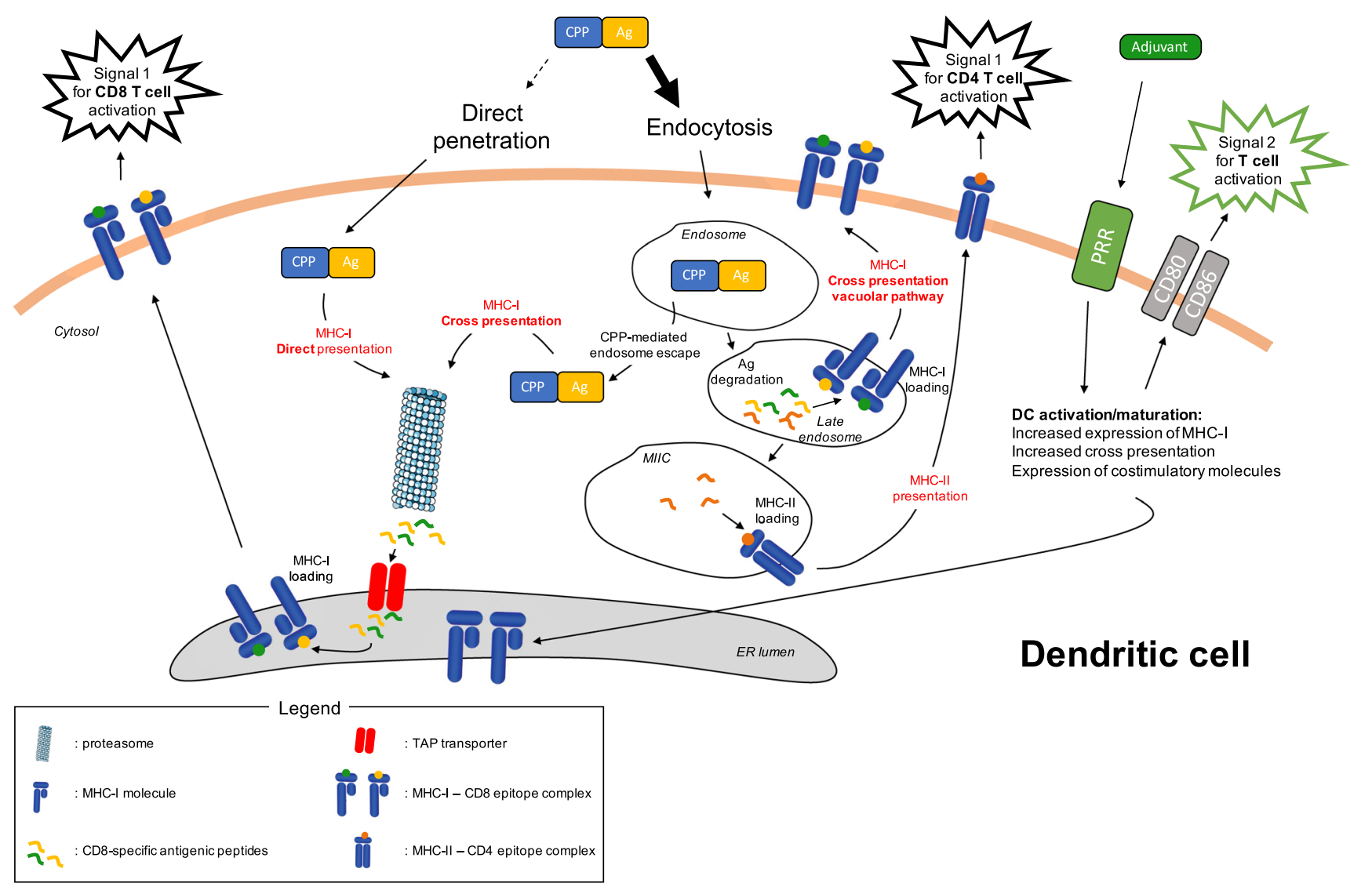

Fig. 1 Ag processing and presentation pathways followed by CPP-Ag in dendritic cells. CPP-Ag mainly enters into the cells via endocytosis, although minor direct penetration across the plasma membrane cannot be ruled out. In the latter situation, antigenic cargo follows the MHC-I direct presentation pathway. Cytosolic antigenic cargo is processed by the proteasome into small antigenic peptides. The transporter associated with antigen processing (TAP) transfers these peptides into the endoplasmic reticulum (ER) where they are loaded onto MHC-I molecules. These complexes are transported to the plasma membrane and deliver the first signal for CD8 T-cell activation through their highly specific interaction with the T-cell receptor (TCR) of cognate CD8 T cells. Endocytosed CPP-Ag can be directed to different pathways. The MHC-I cross presentation pathway is reached by the antigenic cargo after its escape from the endosome due to the intrinsic properties of CPPs. Alternatively, the antigenic cargo can be degraded into small antigenic peptides in the endosome. As MHC-I molecules can be expressed in late endosomes (see Ref. [36]), some of these antigenic peptides can be loaded on these molecules, through the MHC-I cross presentation vacuolar pathway. Antigenic peptides also reach the MIIC compartment where they are loaded on MHC-II molecules that are then transported to the plasma membrane. These complexes deliver the first signal for CD4 T-cell activation through their highly specific interaction with the TCR of cognate CD4 T cells. The role of adjuvant is also depicted. It triggers pattern recognition receptors (PRR), leading to the activation and maturation of dendritic cells. In particular, adjuvant increases the MHC-I cross presentation activity of dendritic cells and also induce the expression of costimulatory molecules that are essential for T-cell activation through the delivery of costimulatory signals. Templates from Servier Medical Art image bank were used to draw this figure stronger OVA-specific OTI T-cell responses in vitro compared to other well-known CPPs including TAT [27]. This increased $\mathrm{Ag}$ presentation could be linked to the fact that LAH4 CPP changes its conformation upon endosome acidification, leading to a facilitated release of antigenic cargo in the cytosol [38]. The CPP KALA adopts an alpha helical conformation at physiological $\mathrm{pH}$ (7.4), enabling it to perform membrane destabilization [39]. Taking advantage of this property, Miura et al. showed that OVA-liposomes fused to KALA exhibited increased MHC-I restricted Ag presentation compared with OVA-liposomes fused to the highly efficient transducer R8 CPP [40]. As a result,
OVA-liposome-KALA vaccination induced higher in vivo CD8 T-cell responses, as revealed by an in vivo cytotoxic assay, and stronger anti-tumor effect in a prophylactic vaccination protocol using EG7 tumor challenge. Surprisingly, an acidic $\mathrm{pH}$ from 6.5 to 5.5 strongly increased the OVAliposome-KALA fusogenic activity, suggesting that this particular construct might destabilize endosome membranes. GV1001 is a telomerase-derived peptide initially used as an MHC class II-restricted epitope in cancer vaccines. Surprisingly, this peptide induced strong in vivo CD4 and CD8 $\mathrm{T}$-cell responses. This unexpected feature relies on the particular cell penetrating property of GV001 enabling it to 
reach the cross presentation machinery. Unlike TAT or other CPPs, the GV1001 cell penetration mechanism involves interaction with extracellular heat shock proteins 70 and 90 , leading to accumulation of this CPP and its cargo in the cytoplasmic compartment [41].

Several studies showed that CPP could be tailored to favor cargo endosome escape. The HA2 domain of influenza haemaglutinin is a $\mathrm{pH}$ sensitive membrane disruptive peptide. Using a TAT-Cre GFP-flox reporter assay, Wadia et al. showed that linking HA2 to the TAT CPP (TAT-HA2) increased the GFP signal observed in transduced cells compared to TAT, due to increased endosomal escape of the CPP-cargo [42]. Similarly, including 10 Histidine moieties to the TAT CPP led to increased endosome escape of the cargo through increased endosome membrane disruption [43]. Finally, Mae et al. showed that $\mathrm{N}$-terminal stearylation of the transportan $10 \mathrm{CPP}$ increased its endosome escape, while it had no effect on penetratin endosomal escape [44]. Regarding ZEBRA-derived CPPs, we used a live cell $\beta$-lactamase reporter assay to monitor cytosolic free protein; $\beta$-lactamase activity with Z12-36KDa was detected with cargo concentrations as low as $100 \mathrm{nM}$, indicating that Z12 efficiently entered the cytosol, although the mechanism was not defined [16].

An alternative vacuolar cross presentation pathway has been described in DCs, which does not require Ag escape from endosomes [36]. In this vacuolar cross presentation pathway, both Ag degradation and epitope loading on MHC-I occur within the endosome and are independent of the proteasome and TAP transporter, two key players in conventional cytosolic cross presentation pathway (Fig. 1). Moreover, it has been demonstrated that protein degradation in endosomes is less efficient in DC compared to other phagocytic cells [45]. Accordingly, several studies reported that CPP-linked Ag processing is TAP- and proteasomeindependent, suggesting that the vacuolar cross presentation pathway might also be used $[46,47]$.

While the complete mechanism of CPP-linked Ag processing and presentation is not fully understood, many of the proof of concept in vivo studies mentioned above showed that the CPP antigenic cargo is efficiently targeted to the MHC-I cross presentation pathway. Indeed, the best readout for efficient targeting of MHC-I cross presentation is the induction of strong Ag-specific CD8 T-cell responses in vivo. Nevertheless, in the context of CPP-based cancer vaccine development, this targeting can and must be optimized to ensure maximal Ag presentation by DCs to T cells.

\section{Multi-epitopic antigenic cargos}

An important characteristic of a CPP-based cancer vaccine is the diversity of its antigenic cargo. Indeed, targeting multiple Ag has several benefits (Fig. 2). First, it might
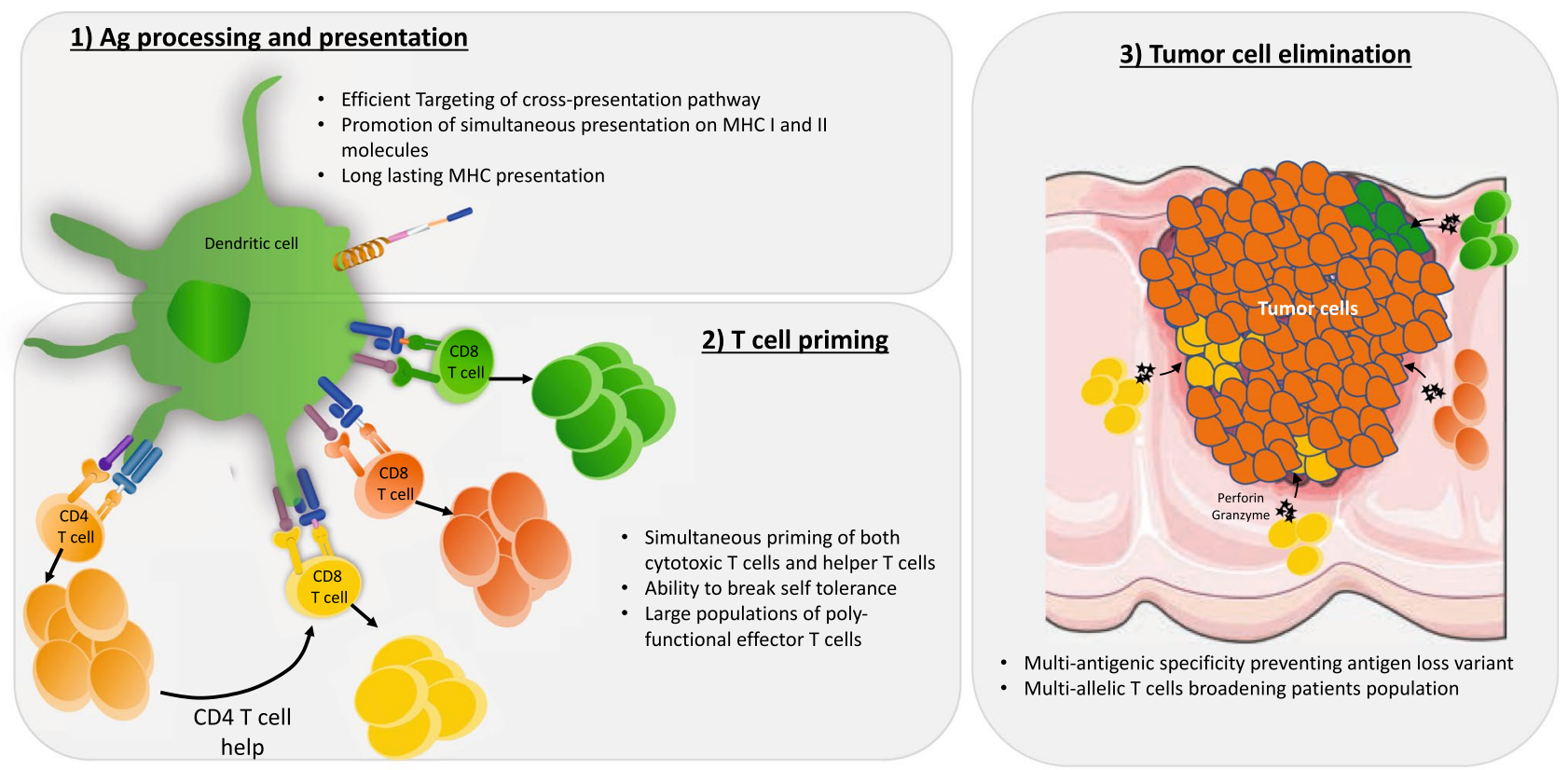

Fig. 2 CPP-based cancer vaccines elicit powerful anti-tumor T-cell responses. CPP vectors allow targeting of multi-epitopic antigenic cargos to processing and presentation machinery for both MHC-I and MHC-II. In the context of appropriate adjuvant, transduced dendritic cells are then able to strongly activate both CD8 and CD4 T cells specific for multiple tumor-derived antigens. The resulting poly-functional effector T-cells migrate to the tumor site, where they eliminate heterogenous tumor cells via cytotoxicity. For each step, the main advantages of CPP-based vaccines are indicated. Templates from Servier Medical Art image bank were used to draw this figure 
limit tumor escape from immune system pressure through the phenomenon of antigen loss variants [48]. Second, it might enlarge the spectra of cancer cells targeted within a single tumor. Indeed, most tumors exhibit heterogeneous Ag expression [49]. Finally, it might enable the same vaccine to be used for wider patient groups by providing epitopes restricted by multiple HLA alleles. We constructed such a multi-epitopic antigenic cargo, including 3 CD8 and 2 CD4specific epitopes, and linked it to the Z12 CPP. In vitro, bone marrow-derived DCs transduced with low amounts $(0.3 \mu \mathrm{M})$ of this construction efficiently stimulated monoclonal CD8 and CD4 T cells of different Ag-specificities; this indicated that each epitope of the cargo was efficiently processed and presented by DCs. In a more physiological polyclonal setting, we demonstrated that vaccination of mice with low amounts of this construct $(10 \mathrm{nmol})$ together with adjuvants induced T-cell immune responses for each of five epitopes, as revealed by MHC-dextramer staining or intracellular cytokine staining [16]. In other studies, TAT was also used as vector for a multi-epitopic cancer vaccine [50, 51]. Multi-epitopic presentation was detected in vitro with $3 \mu \mathrm{M}$ peptide and in vivo with $100 \mu \mathrm{mol}$ of peptide when furinsensitive spacers were added between the epitopes [50, 51]. HSPs derived from cancer cells can form complexes with multiple tumor-derived $\mathrm{Ag}$ that can be up taken by DCs to elicit tumor-specific T-cell immune responses. Nishikawa et al. demonstrated that linking HSP70 to the VP22 CPP dramatically enhances HSP uptake by DCs. Accordingly, in vivo intratumoral electroporation of plasmid encoding VP22-HSP70 in EG7 tumor bearing mice led to strong inhibition of tumor growth. Interestingly, CD8 T cells from vaccinated mice not only lysed OVA-expressing EG7 tumor cells, but also parental EL4 tumor cells that do not express OVA Ag. Thus, combining VP22 to HSP70 generated multiepitopic CD8 T-cell immune responses in vivo [52].

Although CD8 T cells are important for the elimination of cancer cells by cytotoxicity, CD4 T cells are also required for efficacious anti-tumor immunity, through direct effector function, helper activity for CD8 T cells, and enhancement of effector cell infiltration at the tumor site [53]. Therefore, multi-epitopic antigenic cargos linked to CPP stimulating both CD4 and CD8 T cells might also be important to generate a fully functional anti-tumor immune response (Fig. 2). This was directly demonstrated using Zebra-derived CPPs, TAT, and penetratin $[16,22,24,46,54,55]$.

\section{Importance of adjuvants in CPP-based cancer vaccines}

To be fully activated, $\mathrm{T}$ cells have to receive two main signals that are the antigenic signal (signal 1) and the costimulatory signal (signal 2). Moreover, the T-cell response is also shaped by a third signal mediated by cytokines. Signal 1 and signal 2 are delivered by activated mature DCs, as are many cytokines. Triggering of pattern recognition receptors (PRR) on DCs by DAMPs (danger associated molecular pattern) induces increased expression of MHC molecules, costimulatory molecules and cytokines [56] (Fig. 1). Although CPPs are sometimes considered as adjuvants for peptide-based cancer vaccines due to increased intracellular Ag delivery, transduction of immature DC with a CPP-Ag cargo did not induce DC activation and maturation [57-59]. Therefore, CPP-based cancer vaccines must be used in combination with adjuvants to elicit fully functional anti-tumor immune responses.

Several factors, such as limited depot effect and optimal immunopotentiator effect, have to be made regarding the choice of adjuvants used in combination with cancer vaccines [60]. Accordingly, we demonstrated that the choice of adjuvant directly impacts on the immune responses and subsequent anti-tumor effects elicited by CPP-based cancer vaccines. We vaccinated mice with ZEBRA-derived CPPs linked to OVA CD4 and CD8 specific Ag, in combination with Hiltonol (a TLR3 ligand), Pam3CSK4 (a TLR2 ligand) or MPLA (a TLR4 ligand). Efficacy of CPP-adjuvant combinations varied considerably when vaccine-induced CD4 and CD8 T-cell frequencies were measured, which translated into potent therapeutic effect for certain vaccines. Indeed, while all of the adjuvanted vaccines enhanced survival, that induced by Hiltonol or MPLA was particularly striking and more pronounced than that achieved with Pam3CSK4 adjuvant [24]. This study highlights that the choice of adjuvant is a crucial step in the development of a CPP-based cancer vaccine.

The negatively charged double stranded RNA Poly I/C (a less stable equivalent of Hiltonol) is easy to link to cationic CPPs through electrostatic interactions. Transduction of immature DCs with TAT linked to MelanA Ag and Poly $\mathrm{I} / \mathrm{C}$ induced strong DC activation and maturation, as shown by up-regulation of CD83, CD86 and high production of IL-12 [58]. Vaccination of MC38-CEA tumor-bearing mice with TAT-CEA-poly-IC induced a strong decrease of tumor growth compared to TAT-CEA injected mice [57]. In a DC vaccination protocol, Mitsui et al. vaccinated EG7 tumor bearing mice with R9-Ova transduced DCs coupled with three injections of OK432 or LPS (two TLR4 ligands) as adjuvants. They observed that all mice vaccinated with CPP-transduced DCs and adjuvants completely rejected EG7 tumor compared to mice vaccinated with CPP-transduced DCs alone, demonstrating the strong additive effect of adjuvant when used with a CPP-based cancer vaccine [37]. In vivo injection of LAH4-protein with CpG (a TLR9 ligand) increased retention of cargo protein at the site of injection, protein transport to the draining lymph node, and T-cell activation. In a therapeutic vaccination protocol, LAH4-OVA-CpG induced a strong 
anti-tumor effect in B16-OVA tumor bearing mice compared to LAH4-OVA vaccinated mice, as shown by the very strong delay of tumor growth observed [27]. Similar results were observed in a prophylactic vaccination protocol using penetratin-OVA-CpG in a B16 tumor challenge [47].

Cytokines can also be used at adjuvants. In an in vivo vaccination protocol, an IL-15 encoding plasmid linked to TAT CPP and survivin epitope increased the percentage of survivin-specific CD8 T cells with lytic function towards CT26 tumor cells. Moreover, IL-15 adjuvant increased the generation of memory T cells. In CT26 tumor bearing mice, TAT-survivin-IL-15 vaccination was more efficacious than TAT-survivin vaccination [61]. Vaccination of mice with TAT-HPV E7 Ag coupled to a plasmid encoding GM-CSF induced stronger Ag-specific CD8 T-cell responses compared to TAT-E7. This prophylactic vaccination protocol provided a high level of protection, in which $90 \%$ of mice challenged with TC1 tumor cells remained tumor free. In a therapeutic vaccination setting, TAT-E7-GM-CSF provided a strong anti-tumor effect dependent on CD8 T cells and to a lesser extent on CD4 T cells. Moreover, vaccination with TAT-E7-GM-CSF induced more memory CD8 T cells than TAT-E7, conferring long lasting anti-tumor protection [23]. Interestingly, GM-CSF was shown to strongly promote the cross presentation of Ag by DCs [62-64]. Nevertheless, vaccination protocols need to be carefully optimized for this cytokine adjuvant, since either positive or negative effects were reported [65].

In addition to enhancing T-cell responses, adding adjuvants to CPP-based cancer vaccines might also be important to modify the immunosuppressive tumor microenvironment. Indeed, Spinetti et al. recently showed that injection of the TLR7 agonist R848 induced a strong decrease of intratumoral MDSC [66]. The monocytic MDSC subset was the most affected by this treatment. In addition, R848 blocked the immunosuppressive function of this cell population, reducing tumor progression. In a similar manner, injection of an engineered Salmonella typhimurium strain secreting Vibrio vulnificus flagellin B in MC38 tumor bearing mice induced a strong anti-tumor effect, in part through the reprogramming of tumor-associated macrophages to an M1-antitumoral phenotype [67].

\section{Route of administration of CPP-based cancer vaccines}

The main drawback of CPP-based technology is its lack of cell or tissue specificity. As a consequence, systemic injection of CPP leads to integration in multiple cell types (epithelial cells, fibroblasts, leukocytes) within multiple organs $[68,69]$. Strategies have been developed to confer some cell specificity to CPP-based treatments, including coupling to homing peptides or use of activatable CPPs (reviewed in [70]). Although efficacy has been demonstrated, these constructs are mainly used for direct targeting of cancer cells. To the best of our knowledge, no CPP targeting specific immune cell types such as DCs has been developed so far.

Given the crucial role played by DCs in the initiation of T-cell responses, it is of major interest to specifically target vaccine-associated antigens to this cell type. Some of these sentinel cells are positioned in various peripheral sites of the organism, such as skin and mucosal surfaces, where the probability to encounter pathogen is the highest. After Ag capture by these DCs, they start to mature while migrating to draining secondary lymphoid organs to present MHC-Ag complexes to naïve $\mathrm{T}$ cells, leading to their activation [71]. Thus, as for many conventional vaccines, CPP-based vaccines are administered through parenteral injection (either i.d. or s.c.) to maximize the probability of targeting DCs. This route of administration of CPP-based cancer vaccines has been proven to be efficacious, as revealed by strong $\mathrm{Ag}$-specific T-cell responses elicited against various $\mathrm{Ag}$ [21, 24, 47, 72]. An elegant study of Zhang et al. clearly showed that s.c. administration of a CPP-based vaccine efficiently triggers peripheral DCs [27]. Subcutaneous injection of a fluorescent protein coupled to the LAH4 CPP led to increased retention of this protein at the injection site. Strong fluorescence was also detected in inguinal draining lymph nodes. Addition of $\mathrm{CpG}$ adjuvant to this CPPbased vaccine dramatically increased the fluorescence level observed in these lymph nodes. These observations suggest that s.c. injection of CPP-based vaccines efficiently targets skin-resident DCs. Addition of an adjuvant provides the danger signal essential for these targeted DCs to mature and migrate to draining secondary lymphoid organs where they can present captured-Ag to naïve T cells and thus initiate an Ag-specific T-cell response. Accordingly, stimulation of OTI T cells with inguinal lymph node cells from mice vaccinated s.c. with LAH4-OVA-CpG resulted in an almost twofold increase of INF $\gamma$ producing OTI T cells compared to stimulation using lymph node cells from OVA-CpG vaccinated mice.

Using the ANTP CPP coupled to the CD8 epitope SIINFEKL, Schutze-Redelmeier et al. show that the epicutaneous route of vaccine administration induced the strongest SIINFEKL-specific T-cell response, compared to s.c. and i.p. administrations that did not generate detectable responses [73]. Nevertheless, this observation might be due to the fact that this vaccine, compared to the one used by Zhang et al., was administered without adjuvant. In the epicutaneous route, the use of multiple tape-stripping of the skin and acetone treatment might create a local inflammation compensating for this absence of adjuvant.

Other challenging administration routes have been explored to inject cancer vaccines. Intranodal injections 
of RNA-based cancer vaccines showed induction of very strong Ag-specific T-cell responses in mice [74]. However, we found that using this route of administration for a CPPbased therapeutic cancer vaccine generated an $\mathrm{Ag}$-specific immune response of lower magnitude compared to the s.c. route (our unpublished observations).

Some studies suggest that certain immune cells are more prone to be targeted than others by CPP-based vaccines. Schwarze et al. show that in vivo injected TAT- $\beta$ galactosidase construct preferentially accumulated in the red pulp of the spleen, while the white pulp remained largely $\beta$ galactosidase negative [69]. An interesting recent study of Lim et al. shed light on a preferential uptake of CPP-based vaccines by phagocytic cells (DCs and macrophages) compared to other immune cells [75]. In vitro incubation of mouse splenocytes with EGFP coupled to TAT or dNP2 CPPs led to increased EGFP fluorescence in DCs and macrophages compared to lymphocytes. A similar observation was made on splenocytes from mice injected i.v. with these constructs. This preferential targeting of DCs and macrophages by CPP could rely either on their intrinsic phagocytic activity and/or on a different plasma membrane composition of these cells compared to other immune cells, such as heparan sulfate proteoglycans. These molecules are particularly important for the binding and internalization of at least some CPPs [76]. Thus, based on these observations and on the fact that skin is a DC-rich anatomic site, it appears that i.d./s.c. injection might be the most efficacious administration route for CPP-based cancer vaccines.

\section{CPP-based cancer vaccines in clinical trial}

Many cancer vaccines are currently being tested in clinical trials. As DCs are key players in the induction of adaptive immune responses, autologous or HLA-matched Ag-pulsed DCs have been widely used to treat various types of cancers [77]. However, this process is expensive, and to date has offered only modest clinical benefit. Several factors, such as poor MHC class I targeting, short-lived Ag presentation (MHC turnover), or low density of TAAs can be attributed to explain this poor efficacy [78]. In contrast, as discussed above, CPPs represent an outstanding alternative to DCs as a cancer vaccine vector, by increasing Ag uptake, targeting both MHCI and II Ag presentation pathways, by increasing the duration of $\mathrm{Ag}$ presentation, and by increasing the magnitude of Ag-specific immune responses [33, 78]. Nevertheless, a prerequisite is the identification of antigens (TSA or TAA) that can function as tumor-rejection antigens. To date, only a few studies, to our knowledge, have used CPP as a vector for cancer vaccine delivery in a clinical setting. Gliknik Inc. developed a Trojan cancer vaccine, consisting of chemically synthetized long peptides, containing 2 or 3
CD4 and CD8 specific epitopes from either MAGE or HPV $\mathrm{Ag}$, fused to the TAT CPP. A furin-sensitive spacer was added between the epitopes [79]. This approach is based on results showing induction of antigen-specific cytotoxic T lymphocytes after immunization with synthetic long peptides fused to the TAT. In a pilot study, vaccination of five patients suffering from head and neck carcinoma with Trojan vaccine coupled with Montanide and GM-CSF as adjuvants induced antigen-specific CD4 $\mathrm{T}$ cells and $\mathrm{IgG}$ antibodies. Interestingly, Ag-specific CD8 T-cell responses were poorly elicited, perhaps suggesting that antigenic cargo failed to reach the MHC-I cross presentation pathway with this vaccine. None of the patients developed an objective clinical response. Nevertheless, a phase I dose escalation trial was undertaken in a larger cohort of recurrent/metastatic head and neck squamous cell carcinoma patients using the same vaccines [80]. Consistent with their pilot study, induction of Ag-specific CD4 T cells and antibodies was observed in the majority of patients that were vaccinated 4 times. Again, Ag-specific CD8 T-cell responses were not detected. Based on RECIST criteria, no clinical benefit was observed, except for one patient among seven in the MAGE-A3 cohort who experienced stable disease for 10.5 months.

\section{Concluding remarks}

Cell penetrating peptides are very promising tools for the development of new cancer therapies. Conjugating various toxic/antigenic cargos to these short peptides has proven efficacious in specifically targeting and eliminating cancer cells. In the field of cancer vaccines, pre-clinical as well as clinical studies have demonstrated that the use of CPPs as vaccine vectors represents an efficacious strategy to generate strong cancer-specific immune responses. Such an approach is particularly relevant in the case of so-called "cold tumors" that are known to be poorly immunogenic. However, it is challenging to induce an immune response against weakly immunogenic tumors, particularly if the target antigens are TAA to which there is partial immune tolerance. Under immune system pressure, Ag loss variants can arise in the targeted tumor cells, leading to relapse. This phenomenon has been observed in T-cell therapies using adoptive transfer of neo-Ag-specific autologous $\mathrm{T}$ cells in melanoma patients [48]. Thus, the CPP-linked TAAs cargo has to be as diverse as possible. Tumor cells use various mechanisms to escape immune system pressure; among them is the expression of inhibitory immune checkpoints ligands that negatively regulate T-cell function. Antibodies blocking interaction between these ligands and their receptors, such as anti-CTLA4 (ipilimumab), anti-PD1 (nivolumab, pembrolizumab and pidilizumab) and anti-PD-L1, are currently used clinically with outstanding results on a proportion of patients with 
aggressive forms of melanoma and several advanced malignancies [81]. Therefore, immune checkpoint inhibition in combination with CPP-based cancer vaccine immunotherapy represents a promising future direction. Finally, careful choice of adjuvant is mandatory to maximize the magnitude of Ag-specific T-cell responses and the resulting anti-tumor effect elicited by CCP-based cancer vaccines.

\section{Compliance with ethical standards}

Conflict of interest M. Derouazi is the CEO of Amal Therapeutics and has ownership interest (including patents) in Amal Therapeutics and patents related to cell penetrating peptide. P. R. Walker reports receiving joint funding by Swiss Confederation Commission for Technology and Innovation and Amal Therapeutics; has ownership interest in patents related to cell penetrating peptide and is a consultant/advisory board member for Amal Therapeutics.

Open Access This article is distributed under the terms of the Creative Commons Attribution 4.0 International License (http://creativeco mmons.org/licenses/by/4.0/), which permits unrestricted use, distribution, and reproduction in any medium, provided you give appropriate credit to the original author(s) and the source, provide a link to the Creative Commons license, and indicate if changes were made.

\section{References}

1. Frankel AD, Pabo CO (1988) Cellular uptake of the tat protein from human immunodeficiency virus. Cell 55(6):1189-1193

2. Joliot A et al (1991) Antennapedia homeobox peptide regulates neural morphogenesis. Proc Natl Acad Sci USA 88(5):1864-1868

3. Derossi D et al (1994) The third helix of the Antennapedia homeodomain translocates through biological membranes. J Biol Chem 269(14):10444-10450

4. Vives E, Brodin P, Lebleu B (1997) A truncated HIV-1 Tat protein basic domain rapidly translocates through the plasma membrane and accumulates in the cell nucleus. J Biol Chem 272(25):16010-16017

5. Elliott G, O'Hare P (1997) Intercellular trafficking and protein delivery by a herpesvirus structural protein. Cell 88(2):223-233

6. Rothe $\mathrm{R}$ et al (2010) Characterization of the cell-penetrating properties of the Epstein-Barr virus ZEBRA trans-activator. J Biol Chem 285(26):20224-20233

7. Dempsey CE (1990) The actions of melittin on membranes. Biochim Biophys Acta 1031(2):143-161

8. Konno K et al (2000) Structure and biological activities of eumenine mastoparan-AF (EMP-AF), a new mast cell degranulating peptide in the venom of the solitary wasp (Anterhynchium flavomarginatum micado). Toxicon 38(11):1505-1515

9. Esteve E et al (2005) Transduction of the scorpion toxin maurocalcine into cells. Evidence that the toxin crosses the plasma membrane. J Biol Chem 280(13):12833-12839

10. Nascimento FD et al (2007) Crotamine mediates gene delivery into cells through the binding to heparan sulfate proteoglycans. J Biol Chem 282(29):21349-21360

11. Kobayashi S et al (2004) Membrane translocation mechanism of the antimicrobial peptide buforin 2. Biochemistry 43(49):15610-15616
12. Futaki S et al (2001) Arginine-rich peptides. An abundant source of membrane-permeable peptides having potential as carriers for intracellular protein delivery. J Biol Chem 276(8):5836-5840

13. Pooga $\mathrm{M}$ et al (1998) Cell penetration by transportan. FASEB J 12(1):67-77

14. Milletti F (2012) Cell-penetrating peptides: classes, origin, and current landscape. Drug Discov Today 17:850-860

15. Brooks NA et al (2010) Cell-penetrating peptides: application in vaccine delivery. Biochim Biophys Acta 1805(1):25-34

16. Derouazi $\mathrm{M}$ et al (2015) Novel cell-penetrating peptide-based vaccine induces robust $\mathrm{CD}^{+}$and $\mathrm{CD} 8^{+} \mathrm{T}$ cell-mediated antitumor immunity. Cancer Res 75(15):3020-3031

17. Kim DT et al (1997) Introduction of soluble proteins into the MHC class I pathway by conjugation to an HIV tat peptide. J Immunol 159(4):1666-1668

18. Shibagaki N, Udey MC (2002) Dendritic cells transduced with protein antigens induce cytotoxic lymphocytes and elicit antitumor immunity. J Immunol 168(5):2393-2401

19. Apostolopoulos V et al (2006) Delivery of tumor associated antigens to antigen presenting cells using penetratin induces potent immune responses. Vaccine 24(16):3191-3202

20. Pouniotis DS, Apostolopoulos V, Pietersz GA (2006) Penetratin tandemly linked to a CTL peptide induces anti-tumour T-cell responses via a cross-presentation pathway. Immunology 117(3):329-339

21. Bleifuss E et al (2006) The translocation motif of hepatitis B virus improves protein vaccination. Cell Mol Life Sci 63(5):627-635

22. Wang HY et al (2002) Induction of CD4(+) T cell-dependent antitumor immunity by TAT-mediated tumor antigen delivery into dendritic cells. J Clin Investig 109(11):1463-1470

23. Tang $\mathbf{J}$ et al (2012) A novel self-assembled nanoparticle vaccine with HIV-1 Tat(4)(9)(-)(5)(7)/HPV16 E7(4)(9)(-)(5)(7) fusion peptide and GM-CSF DNA elicits potent and prolonged CD8(+) T cell-dependent anti-tumor immunity in mice. Vaccine 30(6):1071-1082

24. Belnoue E et al (2016) Enhancing antitumor immune responses by optimized combinations of cell-penetrating peptide-based vaccines and adjuvants. Mol Ther 24(9):1675-1685

25. Shibagaki N, Udey MC (2003) Dendritic cells transduced with TAT protein transduction domain-containing tyrosinase-related protein 2 vaccinate against murine melanoma. Eur J Immunol $33(4): 850-860$

26. Brooks $\mathrm{N}$ et al (2015) Comparative immunogenicity of a cytotoxic $\mathrm{T}$ cell epitope delivered by penetratin and TAT cell penetrating peptides. Molecules 20(8):14033-14050

27. Zhang TT et al (2012) LAH4 enhances $\mathrm{CD}^{+} \mathrm{T}$ cell immunity of protein/peptide-based vaccines. Vaccine 30(4):784-793

28. Bae MY, Cho NH, Seong SY (2009) Protective anti-tumour immune responses by murine dendritic cells pulsed with recombinant Tat-carcinoembryonic antigen derived from Escherichia coli. Clin Exp Immunol 157(1):128-138

29. Justesen $\mathrm{S}$ et al (2007) Addition of TAT protein transduction domain and GrpE to human $\mathrm{p} 53$ provides soluble fusion proteins that can be transduced into dendritic cells and elicit p53-specific $\mathrm{T}$-cell responses in HLA-A*0201 transgenic mice. Immunology 122(3):326-334

30. Cho HI et al (2007) Enhanced induction of anti-tumor immunity in human and mouse by dendritic cells pulsed with recombinant TAT fused human survivin protein. Cancer Lett 258(2):189-198

31. Yang H, Cho NH, Seong SY (2009) The Tat-conjugated N-terminal region of mucin antigen 1 (MUC1) induces protective immunity against MUC1-expressing tumours. Clin Exp Immunol 158(2):174-185

32. Viehl CT et al (2005) A tat fusion protein-based tumor vaccine for breast cancer. Ann Surg Oncol 12(7):517-525 
33. Wang RF, Wang HY (2002) Enhancement of antitumor immunity by prolonging antigen presentation on dendritic cells. Nat Biotechnol 20(2):149-154

34. Madani $\mathrm{F}$ et al (2011) Mechanisms of cellular uptake of cellpenetrating peptides. J Biophys 2011:414729

35. Guidotti G, Brambilla L, Rossi D (2017) Cell-penetrating peptides: from basic research to clinics. Trends Pharmacol Sci $38: 406-424$

36. Joffre OP et al (2012) Cross-presentation by dendritic cells. Nat Rev Immunol 12(8):557-569

37. Mitsui $\mathrm{H}$ et al (2006) Polyarginine-mediated protein delivery to dendritic cells presents antigen more efficiently onto MHC class I and class II and elicits superior antitumor immunity. J Investig Dermatol 126(8):1804-1812

38. Prongidi-Fix L et al (2007) Self-promoted cellular uptake of peptide/DNA transfection complexes. Biochemistry 46(40):11253-11262

39. Wyman TB et al (1997) Design, synthesis, and characterization of a cationic peptide that binds to nucleic acids and permeabilizes bilayers. Biochemistry 36(10):3008-3017

40. Miura $\mathrm{N}$ et al (2017) Modifying antigen-encapsulating liposomes with KALA facilitates MHC class I antigen presentation and enhances anti-tumor effects. Mol Ther 25:1003-1013

41. Kim H et al (2016) The telomerase-derived anticancer peptide vaccine GV1001 as an extracellular heat shock protein-mediated cell-penetrating peptide. Int J Mol Sci 17(12):E2054

42. Wadia JS, Stan RV, Dowdy SF (2004) Transducible TAT-HA fusogenic peptide enhances escape of TAT-fusion proteins after lipid raft macropinocytosis. Nat Med 10(3):310-315

43. Lo SL, Wang S (2008) An endosomolytic Tat peptide produced by incorporation of histidine and cysteine residues as a nonviral vector for DNA transfection. Biomaterials 29(15):2408-2414

44. Mae M et al (2009) A stearylated CPP for delivery of splice correcting oligonucleotides using a non-covalent co-incubation strategy. J Control Release 134(3):221-227

45. Delamarre L et al (2005) Differential lysosomal proteolysis in antigen-presenting cells determines antigen fate. Science 307(5715):1630-1634

46. Pouniotis D et al (2016) Vaccine delivery by penetratin: mechanism of antigen presentation by dendritic cells. Immunol Res 64(4):887-900

47. Brooks NA et al (2010) A membrane penetrating multiple antigen peptide (MAP) incorporating ovalbumin CD8 epitope induces potent immune responses in mice. Biochim Biophys Acta 1798(12):2286-2295

48. Verdegaal EM et al (2016) Neoantigen landscape dynamics during human melanoma-T cell interactions. Nature 536(7614):91-95

49. Navai SA, Ahmed N (2016) Targeting the tumour profile using broad spectrum chimaeric antigen receptor T-cells. Biochem Soc Trans 44(2):391-396

50. Lu J et al (2004) Multiepitope Trojan antigen peptide vaccines for the induction of antitumor CTL and Th immune responses. J Immunol 172(7):4575-4582

51. Lu J et al (2001) TAP-independent presentation of CTL epitopes by Trojan antigens. J Immunol 166(12):7063-7071

52. Nishikawa $M$ et al (2010) Induction of tumor-specific immune response by gene transfer of Hsp70-cell-penetrating peptide fusion protein to tumors in mice. Mol Ther 18(2):421-428

53. Hoepner S, Walker PR (2013) Getting by with a little help from the right $\mathrm{CD}^{+}{ }^{+} \mathrm{T}$ cells. Oncoimmunology 2(9):e25772

54. Mardani $G$ et al (2016) Protein vaccination with HPV16 E7/ Pep-1 nanoparticles elicits a protective T-helper cell-mediated immune response. IUBMB Life 68(6):459-467

55. Pouniotis DS et al (2011) Whole protein and defined CD8(+) and CD4(+) peptides linked to penetratin targets both MHC class I and II antigen presentation pathways. Immunol Cell Biol 89(8):904-913

56. Gardner A, Ruffell B (2016) Dendritic cells and cancer immunity. Trends Immunol 37(12):855-865

57. Park JS et al (2011) Efficient induction of anti-tumor immunity by a TAT-CEA fusion protein vaccine with poly(I:C) in a murine colorectal tumor model. Vaccine 29(47):8642-8648

58. Haenssle HA et al (2010) Intracellular delivery of major histocompatibility complex class I-binding epitopes: dendritic cells loaded and matured with cationic peptide/poly(I:C) complexes efficiently activate T cells. Exp Dermatol 19(1):19-28

59. Lazoura E, Apostolopoulos V (2005) Insights into peptide-based vaccine design for cancer immunotherapy. Curr Med Chem 12(13): 1481-1494

60. Khong H, Overwijk WW (2016) Adjuvants for peptide-based cancer vaccines. J Immunother Cancer 4:56

61. Yang $\mathrm{Z}$ et al (2008) A novel mimovirus vaccine containing survivin epitope with adjuvant IL-15 induces long-lasting cellular immunity and high antitumor efficiency. Mol Immunol 45(6): 1674-1681

62. Dresch C et al (2012) Development of antigen cross-presentation capacity in dendritic cells. Trends Immunol 33(8):381-388

63. Sluijter BJ et al (2015) Arming the melanoma sentinel lymph node through local administration of CpG-B and GM-CSF: recruitment and activation of BDCA3/CD141(+) dendritic cells and enhanced cross-presentation. Cancer Immunol Res 3(5):495-505

64. Lee SJ et al (2015) Local administration of granulocyte macrophage colony-stimulating factor induces local accumulation of dendritic cells and antigen-specific $\mathrm{CD}^{+} \mathrm{T}$ cells and enhances dendritic cell cross-presentation. Vaccine 33(13):1549-1555

65. Parmiani G et al (2007) Opposite immune functions of GM-CSF administered as vaccine adjuvant in cancer patients. Ann Oncol 18(2):226-232

66. Spinetti T et al (2016) TLR7-based cancer immunotherapy decreases intratumoral myeloid-derived suppressor cells and blocks their immunosuppressive function. Oncoimmunology 5(11):e1230578

67. Zheng JH et al (2017) Two-step enhanced cancer immunotherapy with engineered Salmonella typhimurium secreting heterologous flagellin. Sci Transl Med 9(376):eaak9537

68. Sarko D et al (2010) The pharmacokinetics of cell-penetrating peptides. Mol Pharm 7(6):2224-2231

69. Schwarze SR et al (1999) In vivo protein transduction: delivery of a biologically active protein into the mouse. Science 285(5433):1569-1572

70. Reissmann S (2014) Cell penetration: scope and limitations by the application of cell-penetrating peptides. J Pept Sci 20(10):760-784

71. Merad M et al (2013) The dendritic cell lineage: ontogeny and function of dendritic cells and their subsets in the steady state and the inflamed setting. Annu Rev Immunol 31:563-604

72. Zhao B et al (2013) TAT-mediated gp96 transduction to APCs enhances gp96-induced antiviral and antitumor $\mathrm{T}$ cell responses. Vaccine 31(3):545-552

73. Schutze-Redelmeier MP et al (2004) Antennapedia transduction sequence promotes anti tumour immunity to epicutaneously administered CTL epitopes. Vaccine 22(15-16):1985-1991

74. Bialkowski $L$ et al (2016) Intralymphatic mRNA vaccine induces CD8 T-cell responses that inhibit the growth of mucosally located tumours. Sci Rep 6:22509

75. Lim $S$ et al (2016) Cell type preference of a novel human derived cell-permeable peptide dNP2 and TAT in murine splenic immune cells. PLoS One 11(5):e0155689 
76. Tyagi $\mathrm{M}$ et al (2001) Internalization of HIV-1 tat requires cell surface heparan sulfate proteoglycans. J Biol Chem 276(5):3254-3261

77. Ridgway D (2003) The first 1000 dendritic cell vaccinees. Cancer Investig 21(6):873-886

78. Lim S, Koo JH, Choi JM (2016) Use of cell-penetrating peptides in dendritic cell-based vaccination. Immune Netw 16(1):33-43

79. Voskens CJ et al (2012) Induction of MAGE-A3 and HPV-16 immunity by Trojan vaccines in patients with head and neck carcinoma. Head Neck 34(12):1734-1746
80. Zandberg DP et al (2015) A phase I dose escalation trial of MAGE-A3- and HPV16-specific peptide immunomodulatory vaccines in patients with recurrent/metastatic (RM) squamous cell carcinoma of the head and neck (SCCHN). Cancer Immunol Immunother 64(3):367-379

81. Mahoney KM, Freeman GJ, McDermott DF (2015) The next immune-checkpoint inhibitors: PD-1/PD-L1 blockade in melanoma. Clin Ther 37(4):764-782 